\title{
UN INFORME SOBRE CATALUNYA ELEVADO A CARLOS I EN 1520
}

\author{
F. Udina Martorell
}

A l'Arxiu del Regne de Bèlgica es guarda, entre tanta docu. mentació referent a Carles I, un curiós i confidencial informe so. bre el nostre Principat, i que, sens dubte, enllaça amb textos de l'època que es deurien redactar per a informar els sobirans. Tenim coneixement d'alguna memòria de la primeria del segle XVI que parla d'Espanya, i concretament de la Corona d'Aragó, d'un Manuscrit ${ }^{1}$ datat el $1519^{2}$, és a dir, un any abans del nostre informe ${ }^{3}$.

Sabem que I'any 1519 l'Emperador Carles V es trobava a Molins de Rei: pel que sembla, un secretari seu va demanar una informació sobre el que era el Principat de Catalunya i l'any següent un servent seu i també amic li va trametre una lletra que afortuna. dament s'ha conservat als Arxius del Regne belga4.

1 Tant el coneixement de l'informe que publiquem, com aquestes primeres notes sobre les memòries que s'escrivien per a reforçar els arguments a favor de l'elecció de Carles per a emperador, ho devem al nostre estimat col-lega ale. many, director de l'Institut de Heidelberg, prof. Berthold Beinert.

2 Droysen, Johann Gustav, Zwei Verzeiscbnisse, Kaiser Karls, V, a Abhanduungen der $\mathrm{kgl}$. sachsischen Gesellschaft der Wissenschaften, ABt. III, Bd. 2, Leipzig, 1857, pp. 297.360.

3 BELNERT, BERTHOLD, *Carlos V, señor de muchos reinos, visto a través de los discursos de la coronas, a Cuadermos Hispanoamericanos, t. LXX, núms. 238. 240 , p. 510.

4 Aquest informe que publiquem no tenia realment titol, car es tractrava d'una carta, conviguda ja amb qui l' havia de rebre. Amb lletra posterior es diu en francès Sumari del' Principar de Catalunya. Es guarda als arxius del regne de Bèigica a l'Inventaire du Fonds de l'Audience et ses papiers du Conseil d'Etat, redactat per H. Nelis i sota la cota $1405,(21), 5$. 
L'esmentat informe, que jo he conegut gràcies a la notícia que me'n donà un gran amic alemany $i$ destacat hispanistas, està redactat en català, ocupa sis folis i està escrit amb bon estil, però sense gran estil literari. Dels sis folis es dedica des de l' 1 v. al 2 v., a una breu història del Principat des dels temps de Carlemany fins als del propi emperador, descabdella els fets conegurs per la historiografia de l'època, i és un resum molt breu de la crònica model Turell ${ }^{6}$. És interessant subratllar els judicis sobre la segona muller de Ferran el Catòlic, l'al.lusió a les torbacions, així com la importància que dóna el títol de duc de Girona i Montblanc, vinculat, diu, al comte de Barcelona, que és sobirà del Principat. Fa un gran elogi d'aquell rei, però espera que encara el superarà l'emperador.

A continuació segueix un llarg apartat de tipus geogràfic, que crec que té un gran interès, per la manca de textos d'aquesta època en aquest sentit. Diu que el Principat és una gran terra, i dóna tot seguit les mesures en llengües de Catalunya $i$ per a provar-ho esmenta la grandària del Rosselló, la qual cosa sorprèn un xic. Aleshores hi mescla la divisió de Catalunya en bisbats i els enumera tots, des de l'arquebisbat de Tarragona al darrer bisbat, Elna, explicant que en cadascuna de les ciutats corresponents als bisbats hi ha ebelles y honrades Catedralss. Dóna el nom de cadascun dels titulars de les diòcesis. Parla de les riqueses de dites catedrals i afirma que totes les ciutats i catedrals són del rei, menys la de Tarragona que és meitat del sobirà i meitat de la mitra, o bé la d'Elna que és sols del bisbe.

Aleshores s'entreté a descriure les cases nobiliàries des del duc de Cardona fins al comte de Rialb. Parla de l'almirall de Castella Fadrique Enríquez, que eper causa de la muller que es cathalana, per dot, té el bescomptat de Cabreras, és a dir per Anna vescomtessa de Cabrera. A continuació descriu els vescomtes des del de Canet i Illa fins el de Roda, però diu que seria molt llarga la relació i no continua.

'Regraciem, doncs, al savi hispanista Beinert la notícia i la reiterada invitació que fóssim nosaltres qui la publiquéssim. Així mateix vull agrair al prof. Wiffels, director dels Arxius Nacionals belgues, amabilissim col.lega que he tractat moltes vegades en ocasió de les reunions del Consell Internacional d'Arxius, del qual ell $r$ 'és Secretari Genesal, la gentilesa en trametre' $m$ la fotocoppia de l'esmentat informe. Mercès, dones, ben sinceres a tots dos.

6 TUREL, GABrIEL, Record Barcelona, Ed. Barcino, 1950. Recordi's que el Record' de Turell, com diu el seu editor Enric Bagué, és en conjunt una refundició de les 
Esmenta després els monestirs (encara que entremig hi posa els ducats i les senyories, pertanyents al rei). Cita els de Ripoll, Poblet, Santes Creus, Cuixà i Montserrat i ací fa una al-lusió a les «devotes montanyes» $\mathrm{i}$ a la Moreneta, «molt anomenada per lo mon», on hi ha dotze ermites.

Enumera a continuació les ciutats de Catalunya amb els focs a Balaguer, la darrera que esmenta. Per cert que fa referència a Empúries $\mathrm{i}$ junt amb Tarragona diu que abans eren les emajors ciutats de l'Europas. Després cita les viles (Perpinyà, Puigcerdà, Cervera i Vilafranca del Penedès).

Ens sembla interessant la descripció geogràfica que fa ara del Principat, «que quasi es montanyas i esmenta aleshores les planures més importants: Rosselló, Empordà, el pla de Barcelona, el d'Osona, el d'Urgell i el Camp de Tarragona, citant les ciutats i viles de cadascuna de les planes. \&Tota l'altra part de Catalunya es montanyosa (i aleshores es recorda del pla del Penedès) $\mathrm{i}$ això li dóna peu per a parlar dels castells.

De sobte, com s'esdevé alguna altra vegada, comença a parlar dels llinatges fent-ne una relació molt llarga i situant-los a cadascuna de les ciutats, des de Perpinyà, que és per on comença, fins a Barcelona, on separa els nobles dels cavallers i dels burgesos.

S'acaba l'informe amb un comiat, en el qual el signant, del qual parlarem tot seguit, afirma que és fill de Barcelona i espera que amb aquest informe en tindrà suficient però que està disposat a ampliar-lo si calgués. Signa a la ciutat comtal el dia 21 de gener del 1520 .

El signant m'ha plantejat alguns problemes, com també la persona al qual va adreçada la lletra. El nom de pila d'aquell no ofereix dificultat: Miquel, nom que apareix ben clar, però pel cognom hi figura sols Sp; des d'un primer moment hem suposat que es tracta d'un Spa, malgrat que aquest nom és molt rar. Efectivament l'hem trobat raríssim $^{7} \mathrm{i}$ sols com a topònim una volta, lloc del Berguedà.

Històries e conquestes dels reys d'Aragó e dels comtes de Barcerlona, de Pere Tomic, datat a la vila de Bagà el 1438.

7 Sobre el cognom Spa, o Espà, lectura que ens sembla l'encertada, poc podem dir. Mou en el seu Els llinatges catalans (Raixa, 1959), no el registra, ni tampoc no l'hem trobat en obres nobiliàries. A l'Arxiu de la Corona d'Aragó el trobem registrat a l'Elndex de Riberas i amb grafies d'Espà o Espano (o amb s líquida), l'any 1321 (REG. 220-221, fol. 13), amb referència a Domènec Espà perdonant- 
Es tracta, per contra, d'un Miquel «Scriptor»? No ho creiem, però n'apuntem la possibilitat, majorment quan a la Cancelleria de Carles I hi ha un escrivà que es diu Miquel Agulló.

La trobada a Molins de Rei ens explicaria que Miquel Spa, del qual no hem trobat cap petjada, ocupatia algun càrtec oficial, car en dir que Joan Alemany li va manar d'escriure vol dir que tenia jurisdicció sobre d'ell. No hem trobat, però, cap lligam entre ambdós. Sabem que l'emperador va estar a Molins entre l'octubre de 1419 i el gener de 1520 i durant aquest mes la Cort es trobà ja a Barcelona, que és quan Miquel Spa deuria lliurar-li la carta ${ }^{8}$. A Molins de Rei l'emperador havia rebut la delegació dels prínceps alemanys que li varen comunicar l'haver estat elegit emperador.

lo en un plet entre ell i Guillem de Castellbell, tutor de les filles de Pere Spa (*Spanos) (REG. 2279, fols, 60-61), i el 1662 (REG. 5569, fol. $178 \mathrm{v},-180$ ) en una sentència a favor de la vídua Caterina Spano. Amb aquesta terminació el cognom Spa o Espà, viu aquest encara avui com a cognom, ens recordaria el cognom d'Espanyó (que registra MOL), com a derivat del llatí Hispanione, espanyol, o àdhuc Espanyol, derivat, segons MOLI, com a variant, del llatí vulgar Hispaniolo, variant diu, de Hispanione, espanyol.

8 Vegeu Foronda Y AgULLRA. MANUEi. DE. Estancias y viajes de Carlos V. Madrid, 1914², pp. 152 i ss. També BRANDI, KarL, Kaijer Kan' V, Munich, 1941, T.I. p. 97 i $t$. II, p. 107. Sobre Jean Lalemand, Brandi, op, cif, $t$. I, p. 171 i passim. 
11. Détail sommaire de la Principauté de Catalogne. $1520^{1}$.

Al molt magnific y molt virtuós senyor lo senyor mosen Joan Alaman, secretari de la Çesarea e Catholica Magestad, mon Senyor en sa Cort ${ }^{2}$.

11 v. Senyor. Perque vostra merçe, per son pler, tingua sumaria noticia de aquest nostre Principat de Cathalunya li fas aquesta carta, en la qual per descançar porà veure lo que a Molin de Rey ${ }^{3}$ me menà jo li scrigués.

E primerament ha de saber segons se trove per scriptures ${ }^{4}$ que, apres que lo emperador Karles Maynes fet senyor per sa conquesta del present principat de Cathalunya, ordonà per in perpetuum, que en dit principat fossen nou comptes, nou bescomptes, nou comitors que en altres noms se diuhen nobles, nou marmessos et noucentes cases de homens de paratge. Lo que encare que axi ho ordonas prevengut de la mort non complí, sino que son fill, lo rey Lluis, qui en dit Principat en aquell succehi, ho compli.

E aixi feu los nous comptes seguents, ço es, lo compte de Pallars, lo compte de Urgell, lo compte de Roselló, lo compte de Tarragona, lo compte de Barçelona, lo compte Osona, lo compte de Besalu, lo compte de Empuries, lo compte de Sardanya.

Los nou bescomptes son los seguents: lo bescompte de Villamur, lo bescompte de Agger, lo bescompte de Castellnou, lo bescompte de Scornalbou, lo bescompte de Cardona, lo bescompte de Cabrera, lo bescompte de Bas, lo bescompte de Rocaberti, lo bescompte de Mataplana.

Los nou comitors, qui's diuhen en altres nom nobles, son los seguents: lo noble de Bellera, lo noble de Termes, lo noble Canet, lo no-

${ }^{1}$ L'informe es guarda als Arobives Générales du Royaume de Bélgique, iés un manuscrit intitulat: Détaul sommaire de la Principauté de Catalogne. 1520. inclòs a I'Inventaire du Fonds de l'Audience et de Papiers du Conseil d'Etat, per H. NELIS, sota el número 1405 (21), 5 Notice sommatre sur la Principauté de Calalogne. Com ja hem indicat abans dever el coneixement d'aquest interessant informe al prof. Berthold Beinert, i les facilitats per haver-ne una fotocòpia, sobre la qual hem pogut treballar-hi, al prof. Wiffels, director actualment dels esmentats Arxius belgues. $A$ ambdós els ho regraciem de nou.

${ }^{2}$ Aquesta rúbrica de l'informe no és de la mateixa mà del text dels sis folis de l'informe. perồ și d'una mà contemporània.

'Efectivament, Carles I estigué a Molins de Rei el 1519 i alli hi deuria haver el seu secretari Joan Alemany (o Jean Lallemand), el qual deuria encarregar a Miquel Spa l'informe que li trameté l'any següent, el 1520.

${ }^{4} \mathrm{Tal}$ vegada fa referència als manuscrits que a la seva època es coneixien, però que no hem pogut identificar. Manejà el Tomic? 
ble de Castellet, lo noble de Monclus, lo noble de Santelles, lo noble de Porqueres, que en altre nom vuy son los de Sancta Pau, lo noble de Cervià, lo noble aquest no es en memoria ${ }^{3}$.

Los vervassos son los seguents: lo vervessor de Toralla, lo vervessor de Guimerà, lo vervessor de Muschot, qui vuy en dia se dihen Homs, lo vervessor de Mediona, lo vervessor de Boxadors, lo vervessor de Villadamany, lo vervessor de Bisaura, lo vervessor de Fuxà, aximateix aquest vervessor no's trobe ${ }^{6}$.

Les cases dels nou cents homens de paratge son vuy en dia les cases dels gentills homens antics de Cathalunya. Y diuhen'se homens de paratge per que sempre staven aparellats ab lurs cavalls e armes $/ 2$. per al que per lur senyor sempre los fos menat.

E aquest orde feu dit emperador Karles Maynes y apres complí lo seu fill, lo rey Luis, a honor dels nou ordens dels angells. Y axi per grans temps se mantengue dit Principat ab dit orde.

Lo dit rey Luis donà tots los dits comptats, bescomptats y altres titols a diversos cavallers de lurs exercits y sols ell se retengué per si lo comptat de Barçelona, dant los altres en feu. $\mathrm{E}$ anant'se'n deixà dit comptat de Barçelona a huqui's dia Gaufredo, al qual apres lo dit rey donà lo dit bescomptat [sic], del qual nasqué hun fill qui's dia Grifa Pelos ${ }^{7}$, qui casà ab la filla del compte de Flandes. $\mathrm{E}$ axi durà dit bescomptat [sic] de Barçelona fins al temps del compte Ramon Berenguer, qui casà ab na Petronilla aliter Urranca, filla del rey Remiro, rey de Arago.

E en dit casament se foren pactas que lo regne de Arago y lo Principat de Cathalunya fossen tots units ab una, que lo crit d'e qui anant per a sempre fos de Arago y las insignias e lo/ armas de Cathalunya. Y axi stan vuy en dia, que aragonessos y cathalans criden Arago Sanct Jordi y los cathalans y los aragonessos aporten las insignies e $/ 0 /$ armes de Cathalunya, que son quatre barres vermelles en cap d'or.

E ha durat sucessió fins al temps del rey en Marti, qui mori sens fills ni volgué fer testament. Y aprés mort aquell lo regne de Arago y lo de Valencia y lo Principat de Cathalunya se concordaren de elegir nou persones, tres de cada part, per a que per aquelles fos declarat a qui dits regnes y Principat se squardave; y fou fet de aquexa manera: $y$ les nou persones declararen los dits regnes y Principat sguardar'se a l'infant de Castella, don Ferrando, qui vingué en Arago ab quatre fills, ço es, don Alfonso, don Joan, don Enrich y don Pedro.

5 Evidentment Miquel Espà deuria redactar el seu informe sobre algun manuscrit, com hem dit a la nota anterior, i com ho demostra el fet que digui que no ha romàs memoria del dit nom.

6 De nou la mateixa observació de la nota anterior.

7 Es ben sabuda la freqüent variació del nom de la nostra dinastia de la Casa de Barcelona: Jofre, Guifre, Vifred, Gaufred i Grifa (aquests dos darrers són els que apareixen en el nos. tre manuscrit). 
Y mort lo dit rey don Ferrando succehíli son fill don Alfonso, qui casà ab madama Maria, infanta de Castella, de la qual no agué fills. Y aquest rey don Alfonso $2^{\circ}$ anà en Napols. $\mathrm{Y}$ ab lo gran socos de Barçelona conquistà Napols. I anant's'en per lo cami per mar de Barçelona a Napols, los genovessos lo prengueren y feren ne present al duc de Millà. Y amenant lo a Milà lo duch de Milà li isqué a cami y li aporta les claus de Milà y ua' I molt be de tractar en Milà y apres lo libertà. Y ana's'en en Napols, ahon stigue 22 anys en conquistar Napols i alli mori hun de sos dits germans qui's dia don Pedro /el succeí/.

$\mathrm{Y}$ aquest rey agué hun fill bestart, qui's dia don Ferrando, y morint, en son testament, dexà lo realme de Napols al dit fill bestart y los regnes de Arago a son germa, lo rey don Joan, qui era rey de Navarra per que casà ab la reyna de Navarra. Y axi dit rey don Joan regnà en aquest Principat. Y de la reyna de Navarra agué hun fill, qui's dia Karles. Y apres casà ab una de la casa de l'almirant de Castella ${ }^{8}$, germana que ere de la duquesa vella de Cardona, mare del duch de Cardona, qui viu. Y de aquella agué hun fill don Ferrando. Y a causa que la dita segona muller madastra del dit Princep don Karles sempre lo emulava que volia son fill lo rey don Joan" fos rey, se seguiren en aquest Principat moltes turbacions, pervenint lo dit princep don Karles del reyne de Cicilia, per que ere seu.

Que lo costum de la casa de Arago es aquest: que lo fill primogenit es rey de Cicilia y los cathalans e /o/ barçelonins han'li dada complida promessa de no mancar'li com a tenint'lo per prímogenit. Y anat a son pare lo dit rey don Joan, qui ere en Fraga, lo dit son pare lo feu empresonar y be carçerar; y suplicat per los barçelonins ab moltes y diverses enbaxades may volent' lo libertar fou necessari als dits barçelonins de anar'hi, ma armada, per desliurat dir lur princep primogenit per la promesa li tenien feta, hoc encara per lo que com a faells eren obligats. De modo que lo rey don Joan s'en'anà i lo Karles fou deslliurat, de hon se seguiren moles turbacions. Los huns cathalans declinaven a la voluntat de dit rey don Joan y los altres a la del dit primogenit. $Y$ apres mori lo /3. dit princep don Karles ${ }^{10}$. Y foren tant encesses les turbacions, que no's podien sossegar, ans se seguiren molts desordes en Barçelona, que cercaven reys stranys, pur a la postre Nostre Senyor Deu, qui inlumina les coratges sempre a tot be, disposà que lo rey don Joan, amb lo princep don Fe. rrando, vingué a Pedralbes y aqui stant lo legat, del papa, qui ere aquell que apres fou papa Alexandre ${ }^{11}$ derrer, se tractà bon concordia. Y lo rey don Joan ab son fill entrà en Barçelona y tot lo pessat fou per pessat. Y apres regnà amb molta tranquilitat.

8 Joana Enríquez, filla de Fadrique Enríquez, almirall de Castella, la qual es casà amb Joan (després II) aleshores rei de Navarra, el dia 1 de setembre del 1444.

9 Cal llegir Fernando.

10 Morí el dia 23 d'octubre del 1461.

11 Es tracta d'Alexandre V1. 
Y mort aquell li succehi dit rey don Ferrando, del qual senyor secretari tenim prou noticia. Y si ha fetes coses molt dignes de memoria, no menys ans molt mes speran en Nostre Senyor Deu les fara la Cessarea y Catholica Magestad del Rey nostre Senyor vuy beneventuradament regnant. Y axi fins avuy veu vostra merçe aquest Principat per qui es stat regit y governat.

Mes ha de saber que es huna gran terra aquest Principat, que inclus lo comptat de Rosello y Sardanya te de longitud sinquonta [sic] y set legues grosses com sab vostra merçe que son les legues de Cathalunya y de amplaria 24 legues molt grans.

Ha en dit Principat lo archabisbat de Tarragona, lo bisbat de Barçelona, lo bisbat de Leyde, lo bisbat de Tortosa, lo bisbat de Urgell, lo bisbat de Gerona, lo bisbat de Vich, lo bisbat d'Elna, y en tots aquestos archabisbat y bisbats ultra moltes altres ciutats de gracia ha en quiscun d'ells sa ciutad ab sa Sglessia Katedral molt belles y honrades y son dignitads eccelsiasticas de molt bona y suficient renda. Lo archisbisbat de Tarragona te y possee don Pere de Cardona oncle bestart del duc de Cardona; lo de Leyde te lo germà de Conchillos, secretari del rey; lo de Tortoza, lo cardenal de Tortoza, que es alamany ${ }^{12}$; lo de Urgell, hu de la casa d'Espes, Jo de / 3v. Gerona don Guillem Ramon Boyll, valencià, frare de Sanct Hieronim; lo de Vic, hun valencià, qui fou mestre del fill del compte de Cosetayne ${ }^{13}$, y fou li dat ab intencio que fos de dit fill de dit compte y apres ab lo temps i es restat; lo de Ellna te hun frare castellà de l'orde de Sanct Domingo, qui vuy es enbaxador per lo Rey en Anglaterra. Lo de Barçeiona te lo cardenal Vich, valenciâ, qui ara es stat dat adjunt a don Mari Guarcia, aragones, qui es molt vell ${ }^{4}$.

Vuy en dia totes les dites dignitats seculars de dits comptats y bescomtars son reduides a poques persones. Y per ço ha de saber que vuy en dia totes les ciutats y Katedrals y de gracia en lo present Principat de Cathalunya son del rey, acepto [sic] Tarragona, que es mixta ab lo rey y archabisbe, hi Elna, que es del bisbe, y algunes altres que los ecclesiastics y tenen alguna cosa.

Apres del rey, gran vuy en dia no y ha sino lo duch de Cardona, qui's creu te de renda vint milia ducats y te molts vasalls y bons y grans terres; ell te lo comptat de Prades, lo marquessat de Pallars, la baronia d'Entenza, que es gran cosa, lo ducat de Cardona y moltes altres terres y baro-

12 Es Adrià d'Utuecht (1459-1523), sacerdot i degà de Sant Pau de Lovaina. Havia estat preceptor del jove princep Carles. Va representar els seus interessos, quan Carles era duc de Borzonya, prob del seu avi matern Ferran el Catòlic, des de 1515.

El 1517 Caries el ptesentả a Lleó $\mathrm{X}$ per al bisbat de Tortosa. Per influència de l'emperador fou elegit papa, amb el nom d'Alexandre VI, el 1522; i mori el 1523.

13 Es tracta de Joan IV de Tormo (1511-1553).

14 Sembla que es tracta de Guillem Ramon de Vic, cardenal (1521 a 1525), malgrat que l'any 1520, data de l'informe, no era bisbe encara. 
nies. Mes hi ha lo duch de Segorb [sic] qui te en aquest Principat lo comptat de Castelló de Empuries, que es cosa honrada. Mes, lo almirant de Castella, qui per causa de la muller, que es cathalana ${ }^{15}$, per dot, te lo bescomptat de Cabrera, lo bescomptat de Bas, que es una gran cosa y rica y de molt vasals y de moltes viles gentils a la marina entre Gerona y Barçelona. La reyna dona Germana, reyna de Arago etc., te lo bescomptat de Castellbo y la baronia de Rialp y de la valla de Assua (?), y que ha pessar mill y doscents 4. vasals. Don Francisco de Lluna, aragones, te lo marquessat de Camarassa.

Apres hi ha molts bescomptes; son: lo bescompte de Evoll, lo bescompte de Canet, lo bescompte d'Illa, lo bescompre de Rocaberti, lo bescompte de Roda. Mes hi ha lo compte de Palamos, qui es don Ramon de Cardona. De barons, molts: lo baro de Erill, lo baro de Bellera, que pos ha es mort, lo baro de Santelles, qui es lo compte de Quirra, lo baro Senesterra, lo baro de Lagostera, lo baro de Castelldefels y molts altres, que seya cosa larga de recitar.

Mes ha de saber que en aquest Principat ha molts abbats, molts priors y moltes altres dignitats ecclesiassticas y signantment hi ha moltes abbadies de Sanct Benet y de Sanct Bernat. Y dites persones ecclessiastiques tenen moltes baronies, moltes locs, perque antigament los catalans, quant morien, sens fills, dexaven tots lurs locs a las iglessies. E senyaladament Vich ha hon monastir de Ripoll, bella cosa, ahon antigament si soterraven tots los comptes de Barçelona. Perque ha de entendre que encara que aquest Principat se digua principat de Cathalunya no y ha titol de princep de Cathalunya, mes lo titol es de compte de Barçelona y lo qui es compte de Barcelona, es senyor del principat de Catalunya ${ }^{16}$.

Mes ha de saber que en Cathalunya ha mes dos ducats y una senyoria, ço es, lo ducat de Gerona y lo ducat de Monblanc, qui vuy en dia son del rey, mes avent lo rey fill, lo primogenit se diu duch de Gerona y duch de Monblanc, y senyor de Balaguer. Mes per les comptesas de Barçelona / $4 \mathrm{v}$, ha dos cambres de aquestos locs, ço es, de Tarraga, de Villagrassa y de Sabadel, que sempre son cambres de la reyna, que en Cathalunya son com a comptessa de Barçelona ${ }^{17}$.

Mes hi ha hun bellissim monastir de l'orde de Sanct Bernat, qui's diu Poblet y alli se acustumen soterar los reys de Arago. Y aquest monastir te molts locs, villes y castells y es riquissim. Apres hi ha hun altre monestir de Santas Creus; lo monastir de Sanct Miquel de Cuxa, lo mo-

15Es tefereix a Anna (que moni el 1565). filla de Joan i Anna; la teferida Anna esmentada en primer lloc era comtessa de Modica i d'Osona, vescomtessa de Cabrera i de Bas, que es casã, essent encara impúber, el 1515 amb Lluís Enríquez Girón, comte de Melgar i due de Medina de Rioseco.

16 Aclariment molt pertinent per a un estranger.

17 Clara exposició dels títols del primogènit i de la comtessa de Barcelona. 
nastir de Sancht Culgat y molts altres asenyallats monestits. Majorment hi ha unes molt devotes montanyes, que's diuhen de Montserrat, ahon ha hun monastir devot de observança de l'orde de Sanct Benet y aqui es la iglesia de Nostra Senyora de Monserrat molt anomenada per lo mon; en les montanyes ha 12 hermitans.

Les ciutats principals per orde venint de majors a menors son: Barçelona, ahon ha de set en vuyt milia focs; Leyde y deu haver mil focs; Gerona, que es del mateix tamany, empero creex cada dia y se spera esser una gran ciutat ${ }^{18}$; Tarragona, que es de vuyt cents focs, empero antigament ella y la ciutad de Empuries, que era en Cathalunya, que ara es derruida, eren las majors ciutads de la Europa; Tortoza, que es de la mateixa granaria; Vic, que es de sis cents o set cents focs; Ellina que es de trecens focs y Urgell aiximateix; Balaguer es ciutad de gran [aria], es de trecens en quatrecents focs; Manresa, que aximateix es ciutad de gran [aria], es del tamany ${ }^{19}$,

Viles son les seguents: Perpinya, que es de tres milia en quatre milia focs; Puigserda, que es de quatracents en sincents focs; Cervera, que es de sincents cases; Vilafranca de Panades, que es de trecents o quatrecents focs y de docents, trecents focs moltes altres viles; de cent infinides locs, de menys focs seria lare de recitar.

Han mes en Cathalunya, perque 15. quasi es montanyàs, sols los plans mes principals seguents: ço es, lo pla de Rosello, ahon sta Perpinya, Elna y moltes villes, lo pla Empurda, ahon es Gerona, Figueres, Perellada, Castelló de Empuries, Bascara, Roses, Cadaques, Torroella de Montgrí, La Bisbal y moltes altres viles y locs y es gentill terra; lo pla de Barçelona encare, que es per ahon en Barçelona y molts Jogarets; lo pla de Ossona, ahon sta Vich y moltes viles y locs; lo pla de Urgell, ahon sta Leyde, Cervera, Tarraga, Bellpuig, Anglassola, Balaguet, Agramunt, Arbeca y moltes altres viles y locs; lo pla del Camp de Tarragona, que es hun jardí; ha Tarragona, Cambrills, Montoig, Reus, Vallmoll, Valls y moltes altres grosses villes. Tota l'altre part de Cathalunya es montanyossa, ab alguns pocs plans, y non oblit lo pla de Vilafranca de Penades, ahon es Vilafranca, l'Arbos, al Venrell y moltes altres villes y locs.

En los locs montanyossos ha de beles viles y moles y bons castells. E los principals castells de Cathalunya son: de Salses, de Perpinya, dita Bellaguarda, de Belver, de Leyda, de Tortoza, de Cervera, de Ciutat de Castellbo y altres. $Y$ aquestos son reals y de guarda salvo lo de Castellibo qui es de la reyna, com dait es dit. Es hi mes lo castell de Monsoriu, qui es de guarda, mes el de l'almirant de Castella y molts altres castells en locs moit aspres.

18 Curiosa observació sobre Girona, que no sabem a què obecix.

19 El Fogatge de 1515 de l'Arxiu de la Corona d'Aragó pogué ésser una de les fonts de Mi. quel Espa. 
Entre los altres principals linatges hi homens tenguts en stima de Cathalunya me ocorra ara pesant [sic] per locs com en Perpinya; los Homs, los Taquins, los Blancs, los Alberts, los Vivers, los Ortafans, los Ballarons y molts altres; en Gerona: los Çarrieres, los Margarits, los Sitjars, los Ceguyoles, los Sanctmartins, los Biures, los Agullanes, los Terrades y molts altres; / $5 v$. los Monradons, los Monsunisses, los Gurbs, los Malles, los Vilas, y molts altres; en Urgell: los Tragons, los Aguillars, los Lordats; en Tortoza: Iso Olivers, los Botellets, los Genestas y molts altres; en Tarragona: los Castellets, los Ysarts, los Monpalat, los Tamarits, los Caportellas, y molts altres; en Leyde: los Riquers, los Remolins, los Pous, los Gralles, los Monsuars, los Valls, los Cardoners, los Despes y molts altres; en Balaguer: los Vedunyes y sos gendres los Spitals y molts altres; en Manresa: los Pagueres, los Gaves, y molts altres; en Çervera: los Gilaberts, los Altarribes, los Aymerics, los Ceplanes y molts altres; en Barçelona: de los nobles: los de la casa de Cardona, los de la casa de Rocaberti, los de Canet, los de Requesens, los Castellets, los Ycarts, los Balleres, los Muncades, los Erills, los de Castro y de Pinos, los Cruilles, los de So, los Cervellons, los de Queralt, los de Sentelles, los Rebolledors, los Gosse $\mathrm{G}^{20}$ y molts altres; de cavallers y gentills homens; los Santeliments, los Durals, los Torrens, los Pagueres, los Corberas, los Gralles, los Golbers $^{21}$, los Ferres, los Turels, los Monbuys, los Bertrans de Gelida; los burguessos: los Stalrics, los Villatortas, los Clasquerins, los Çalbans, los Sempmenats, los Valls, Jos Olivers, Jos Guimerans, los Boxados, los Blanes, los Alamanys, los Torrellas y infinits altres ${ }^{22}$; dels ciutadans: los Maymons, los Marquets, los Çepiles, los Romeus, los Torrents, los Lulls, los Busots, los Vallsecas, los Junyents, los Aguillars, los Dusays, los Massaners, los Sancliments, los Bestides, los Giberts, los Sanctjust, los Ballesters y molts altres que seria cosa largua d'scriure'lls com axi en moltes altres parts de Cathalunya son infinits locs que per ara no crec recitar.

Con suplich a vostra merçe age prou per aquesta largua carta del que dalt sta recitat, que lo restant sempre que vulla le' $y$ dira aquest, qui fa fi en Barçelona, de hon natural es, a 21 de janer $1520^{23}$.

De vostra merçe, molt cert servidor.

Miquel Spa ${ }^{24}$

20 Segurament vol dir Josses, del cognom Jossa.

21 Suposem que vol dir Gualbes.

22 No són massa correctes les classificacions que fa l'autor; vegi's, per exemple, com col-lo-

ca els Setmenat i els Boxadors.

23 Un dels pocs detalls que sabem de la vida de Miquel Espà.

24 No hem sabut llegir més que Spa Espà, ja que les lletres $S$ i p no ens porten a cap més cognom. Sobre aquest ja en parlem al comentari que va davant del rext que publiquem. 